\title{
Spectrum of signless 1-Laplacian on simplicial complexes
}

\author{
Xin Luo* \\ School of Mathematics \\ Hunan University \\ Changsha, P.R. China \\ Academy of Mathematics and Systems Science \\ Chinese Academy of Sciences \\ Beijing, P.R. China \\ xinlnew@163.com xinluo@amss.ac.cn \\ Dong Zhang ${ }^{\dagger}$ \\ School of Mathematical Sciences \\ Peking University \\ Beijing, P.R. China \\ 13699289001@163.com dongzhangmath@gmail.com \\ Submitted: Aug 18, 2019; Accepted: Apr 13, 2020; Published: May 29, 2020 \\ (C) The authors. Released under the CC BY-ND license (International 4.0).
}

\begin{abstract}
We introduce the signless 1-Laplacian and the dual Cheeger constant on simplicial complexes. The connection of its spectrum to the combinatorial properties like independence number, chromatic number and dual Cheeger constant is investigated. Our estimates can be comparable to Hoffman's bounds on Laplacian eigenvalues of simplicial complexes. An interesting inequality involving multiplicity of the largest eigenvalue, independence number and chromatic number is provided, which could be regarded as a variant version of Lovász sandwich theorem. Also, the behavior of 1-Laplacian under the topological operations of wedge and duplication of motifs is studied. The Courant nodal domain theorem in spectral theory is extended to the setting of signless 1-Laplacian on complexes.
\end{abstract}

Mathematics Subject Classifications: 05E45, 47J10, 49R05

*Supported by China Postdoctoral Science Foundation (No. 2019M660829)

†Supported by China Postdoctoral Science Foundation (No. BX201700009) 


\section{Introduction}

It is widely known that the discrete Laplacian on graphs has been studied for a long history, and B. Eckmann has generalized it to simplicial complexes which encodes the topological and combinatorial data [10]. Some recent remarkable results on discrete Laplacian for simplicial complexes involve lots of algebraic and geometric aspects for complexes, which are applied in the study of expansion, mixing, colorings, random walks, geometric and topological overlap $[12,13,2,11,24]$. Other relevant operators like adjacency operator and Hecke operator (see [9]), as well as signless Laplacians [18], are systematically studied with many interesting applications [17, 20, 21, 22].

On the other hand, the (signless) 1-Laplacian on graph has been also investigated systematically, see $[16,5,6,7,4]$. For (signless) 1-Laplacian, the (dual) Cheeger inequality turns out to be an equality which is rather different from the discrete Laplacian. Besides, the multiplicity of the maximum eigenvalue possesses strong relations with the independence number and chromatic number [27]. Due to these exciting results, 1-Laplacian seems to perform better on combinatorial properties of graphs than Laplacian. In a sense, the (signless) 1-Laplacian provides a zero-homogenous spectral theory on graph, with which some advantages on graphic features are founded.

To some extent, the previous explorations indicate that finding a zero-homogenous spectral theory for a combinatorial object may bring new discoveries and better discrete features - this is the big picture behind our researches. In the present paper, the motivation is to realize a zero-homogenous spectral theory for simplicial complexes, which is a preliminary attempt under the big picture. As a good starting point, signless 1-Laplacian seems to be a suitable and simple choice along this direction - because we don't need to consider the routine orientations of a simplical complex when we use signless 1-Laplacian rather than 1-Laplacian.

In the present paper, we will mainly focus on bridging the spectrum of signless 1Laplacian and some combinatorial data of complexes including the independence number, chromatic number and clique covering number, etc. The organization of this paper is as follows. Some basic definitions and a preliminary result involving the spectrum of signless 1-Laplacian on complexes is shown in the following subsections. The Courant nodal domain theorem (see Theorem 5) is investigated in Section 2. One of the main parts is Section 3 where one can find strong and close relations between the spectrum of signless 1-Laplacian and some combinatorial parameters of complexes (see Theorems 7, 10 and 13). Finally, we study the spectrum for 1-Laplacian on complexes constructed via wedges and duplication of motifs in Section 4.

We highlight here that both Theorem 7 and Theorem 10 provide Hoffman-type bounds on independence number and chromatic number of a simplicial complex, which can be comparable to the classical versions of Hoffman's bounds [2, 11, 14, 15, 23]. Meanwhile, Theorem 10 bridges the 1-Laplacian spectrum, the chromatic number and the dual Cheeger constant. And interestingly, Theorem 13 shows a variant analog of the Lovász

Sandwich theorem $\alpha \leqslant \Theta \leqslant \theta \leqslant \kappa$ with $\Theta$ the Shannon capacity and $\theta$ the Lovász theta number [2]. 


\section{$1.1 \quad$ Preliminary and definitions}

An abstract simplicial complex $K=(V, S)$ on a finite vertex set $V$ is a collection of subsets of $V$ that is closed under inclusion, i.e. $F^{\prime} \subset F \in S \Rightarrow F^{\prime} \in S$. A $d$-face is an element of cardinality $(d+1)$ in $S$. The collection of all $d$-faces of the simplicial complex $K$ is denoted by $S_{d}(K)$ and the number of all $d$-faces is denoted by $\# S_{d}(K)$ or $\left|S_{d}(K)\right|{ }^{1}$ Assume that $\bar{F}=\left\{v_{0}, v_{1}, \ldots, v_{d+1}\right\}$ is a $(d+1)$-face of a complex $K$, then $F_{i}=\left\{v_{0}, \ldots, \hat{v}_{i}, \ldots, v_{d+1}\right\}$ is a $d$-face of $\bar{F}$ which can be denoted by $F_{i} \prec \bar{F}$. The boundary set $\partial \bar{F}$ can be divided into $(d+2)$ boundary faces, $\left\{F_{i}\right\}_{0 \leqslant i \leqslant d+1}$. For two $d$-faces $F_{i}$ and $F_{j}$ sharing a $(d-1)$-face, we say they are $(d-1)$-down neighbours and denote by $F_{i} \stackrel{\text { down }}{\sim} F_{j}$. Similarly, for two $d$-faces $F_{i}$ and $F_{j}$ which are boundaries of a $(d+1)$-simplex, we use the term of $(d+1)$-up neighbours and write as $F_{i} \stackrel{\text { up }}{\sim} F_{j}$.

According to the combinatorial structure related to up or down adjacency, we define signless up 1-Laplacian and signless down 1-Laplacian respectively. Henceforth, we omit the word 'signless' for simplicity, that is, we will use (up and down) 1-Laplacian instead of signless 1-Laplacian.

Let $p=\# S_{d+1}(K), q=\# S_{d}(K)$ and $l=\# S_{d-1}(K)$. The incidence matrix corresponding to $S_{d+1}(K)$ and $S_{d}(K)$ is defined by $B_{d}^{u p}=\left(b_{F_{i} F_{j}}^{u p}\right)_{p \times q}$, where

$$
b_{\bar{F}_{i} F_{j}}^{u p}= \begin{cases}1, & \text { if } F_{j} \in \partial \bar{F}_{i}, \\ 0, & \text { if } F_{j} \notin \partial \bar{F}_{i} .\end{cases}
$$

Similarly, the incidence matrix corresponding to $S_{d}(K)$ and $S_{d-1}(K)$ is $B_{d}^{\text {down }}=\left(b_{E_{j} F_{i}}^{\text {down }}\right)_{l \times q}$, where

$$
b_{E_{j} F_{i}}^{\text {down }}= \begin{cases}1, & \text { if } E_{j} \in \partial F_{i}, \\ 0, & \text { if } E_{j} \notin \partial F_{i} .\end{cases}
$$

For any $f=\left(f_{1}, \cdots, f_{q}\right) \in \mathbb{R}^{q}$, define the set valued mapping Sgn $: \mathbb{R}^{q} \rightarrow 2^{\mathbb{R}^{q}}$ by

$$
\operatorname{Sgn}(f)=\left\{\left(v_{1}, \cdots, v_{q}\right)^{T} \in \mathbb{R}^{q}: v_{i} \in \operatorname{Sgn}\left(f_{i}\right), i=1, \cdots, q\right\}
$$

i.e.,

$$
\operatorname{Sgn}(f)=\operatorname{Sgn}\left(f_{1}\right) \times \operatorname{Sgn}\left(f_{2}\right) \times \cdots \times \operatorname{Sgn}\left(f_{q}\right)
$$

where $2^{\mathbb{R}^{q}}$ is the power set of $\mathbb{R}^{q}$ and

$$
\operatorname{Sgn}(t)= \begin{cases}\{1\}, & \text { if } t>0 \\ \{-1\}, & \text { if } t<0 \\ {[-1,1],} & \text { if } t=0\end{cases}
$$

Then define the up and down 1-Laplacian respectively as follows:

$$
\begin{aligned}
\Delta_{1, d}^{\text {up }} f & =\left(B_{d}^{\text {up }}\right)^{\top} \operatorname{Sgn}\left(B_{d}^{\text {up }} f\right), \\
\Delta_{1, d}^{\text {down }} f & =\left(B_{d}^{\text {down }}\right)^{\top} \operatorname{Sgn}\left(B_{d}^{\text {down }} f\right),
\end{aligned}
$$

\footnotetext{
${ }^{1}$ Sometimes, we will also use $|\cdot|$ to denote the absolute value. While the readers could easily distinguish the meanings.
} 
where $f=\left(f_{1}, f_{2}, \cdots, f_{q}\right)$ is a real $q$-dimensional vector with components corresponding to the function values on every $d$-face, i.e., $f_{i}:=f\left(F_{i}\right)$. And $B^{T} \operatorname{Sgn}(B f)$ is defined as $\left\{B^{T} g: g \in \operatorname{Sgn}(B f)\right\}$ in which $B=B_{d}^{u p}$ or $B_{d}^{\text {down }}$. Hereafter, for convenience, with some abuse of notation, $f_{i}$ also represents the $d$-face with subscript $i$.

Remark 1. If we remove the 'Sgn' in the definition of (signless) 1-Laplacians $\Delta_{1, d}^{u p}$ and $\Delta_{1, d}^{\text {down }}$, then we can get the signless Laplacian defined on a simplicial complex [18]. This can be verified in the following way: note that $\left(B_{d}^{u p}\right)^{\top} B_{d}^{u p} f=\left(D_{d}^{u p}+A_{d}^{u p}\right) f$, where $D_{d}^{u p}=\operatorname{diag}\left(\operatorname{deg}_{1}^{u p}, \ldots, \operatorname{deg}_{q}^{u p}\right)$ with $\operatorname{deg}_{i}^{u p}$ is the number of $(d+1)$-faces containing $f_{i}$ in boundary, and $A_{d}^{u p}=\left(a_{j j^{\prime}}\right)_{q \times q}$ is the adjacent matrix defined as

$$
a_{j j^{\prime}}= \begin{cases}1, & \text { if } F_{j} \cup F_{j^{\prime}} \in S_{d+1}(K) \\ 0, & \text { otherwise }\end{cases}
$$

If we adopt the more general form

$$
b_{\bar{F}_{i} F_{j}}^{u p}= \begin{cases}\sqrt{w_{i}}, & \text { if } F_{j} \in \partial \bar{F}_{i}, \\ 0, & \text { if } F_{j} \notin \partial \bar{F}_{i} .\end{cases}
$$

where $w_{i}$ is a nonnegative weight on $\bar{F}_{i} \in S_{d+1}(K)$, then by standard normalization, we arrive at the (normalized) signless Laplacian

$$
\frac{1}{d+2}\left(D_{d}^{u p}\right)^{-1 / 2}\left(B_{d}^{u p}\right)^{\top} B_{d}^{u p}\left(D_{d}^{u p}\right)^{-1 / 2}=\frac{1}{d+2}\left(I+\left(D_{d}^{u p}\right)^{-1 / 2} A_{d}^{u p}\left(D_{d}^{u p}\right)^{-1 / 2}\right),
$$

which is called the upper random walk on $d$-simplices defined by a transition probability matrix (Definition 3.1 in [18]). For the lower case in [18], we have a similar discussion as above.

Definition 1 (eigenvalue problem for up 1-Laplacian). A pair $(\mu, f) \in \mathbb{R} \times\left(\mathbb{R}^{q} \backslash\{0\}\right)$ is called an eigenpair of the up 1-Laplacian $\Delta_{1, d}^{u p}$, if

$$
0 \in \Delta_{1, d}^{u p} f-\mu D^{u p} \operatorname{Sgn}(f) \quad\left(\text { or } \mu D^{u p} \operatorname{Sgn}(f) \bigcap \Delta_{1, d}^{u p} f \neq \varnothing\right),
$$

where $D^{u p}=\operatorname{diag}\left(\operatorname{deg}_{1}^{u p}, \ldots, \operatorname{deg}_{q}^{u p}\right)$ and $\operatorname{deg}_{i}^{u p}$ is the number of $(d+1)$-faces containing $f_{i}$ in boundary. It should be noted that $\Delta_{1, d}^{u p} f-\mu D^{u p} \operatorname{Sgn}(f)$ means the Minkowski summation of the vector sets $\Delta_{1, d}^{u p} f$ and $-\mu D^{u p} \operatorname{Sgn}(f)$.

A pair $(\mu, f) \in \mathbb{R} \times\left(\mathbb{R}^{q} \backslash\{0\}\right)$ is called an unnormalized eigenpair of the up 1-Laplacian $\Delta_{1, d}^{u p}$, if

$$
0 \in \Delta_{1, d}^{u p} f-\mu \operatorname{Sgn}(f) \quad\left(\text { or } \mu \operatorname{Sgn}(f) \bigcap \Delta_{1, d}^{u p} f \neq \varnothing\right) .
$$

Definition 2 (eigenvalue problem for down 1-Laplacian). A pair $(\mu, f) \in \mathbb{R} \times\left(\mathbb{R}^{q} \backslash\{0\}\right)$ is called an eigenpair of the down 1-Laplacian $\Delta_{1, d}^{\text {down }}$, if

$$
0 \in \Delta_{1, d}^{\text {down }} f-\mu D^{\text {down }} \operatorname{Sgn}(f) \quad\left(\text { or } \mu D^{\text {down }} \operatorname{Sgn}(f) \bigcap \Delta_{1, d}^{\text {down }} f \neq \varnothing\right),
$$


where $D^{\text {down }}=\operatorname{diag}\left(\operatorname{deg}_{1}^{\text {down }}, \ldots, \operatorname{deg}_{q}^{\text {down }}\right)$ and $\operatorname{deg}_{i}^{\text {down }}=(d+1)$, i.e., the number of $(d-1)$-faces of $f_{i}$.

Similarly, a pair $(\mu, f) \in \mathbb{R} \times\left(\mathbb{R}^{q} \backslash\{0\}\right)$ is called an unnormalized eigenpair of the down 1-Laplacian $\Delta_{1, d}^{\text {down }}$, if

$$
0 \in \Delta_{1, d}^{\text {down }} f-\mu \operatorname{Sgn}(f) \quad\left(\text { or } \mu \operatorname{Sgn}(f) \bigcap \Delta_{1, d}^{\text {down }} f \neq \varnothing\right) .
$$

Direct calculation shows

$$
\left(\Delta_{1, d}^{u p} f\right)_{i}=\left\{\sum_{j_{1}, \ldots, j_{d+1}} z_{i j_{1} \cdots j_{d+1}} \mid z_{i j_{1} \cdots j_{d+1}} \in \operatorname{Sgn}\left(f_{i}+f_{j_{1}}+\cdots+f_{j_{d+1}}\right)\right\}
$$

where the summation $\sum_{j_{1}, \ldots, j_{d+1}} z_{i j_{1} \cdots j_{d+1}}$ is taken over all $d$-faces that $f_{j_{1}}, \cdots, f_{j_{d+1}}$ and $f_{i}$ are in a same $(d+1)$-face. Moreover, $z_{i j_{1} \cdots j_{d+1}}$ is symmetric on its indices. In coordinate form, the eigenvalue problem (1) for up 1-Laplacian is to solve $\mu \in \mathbb{R}$ and $f \in \mathbb{R}^{q} \backslash\{0\}$ such that there exists $z_{i j_{1} \cdots j_{d+1}}$ satisfying

$$
\sum_{j_{1}, \ldots, j_{d+1}} z_{i j_{1} \cdots j_{d+1}} \in \mu \operatorname{deg}_{i}^{u p} \operatorname{Sgn}\left(f_{i}\right), i=1,2, \ldots, q
$$

Similarly, the coordinate form of down 1-Laplacian reads as

$$
\left(\Delta_{1, d}^{\text {down }} f\right)_{i}=\left\{\sum_{i_{1}, \cdots, i_{m}} z_{i i_{1} \cdots i_{m}} \mid z_{i i_{1} \cdots i_{m}} \in \operatorname{Sgn}\left(f_{i}+f_{i_{1}}+\cdots+f_{i_{m}}\right)\right\}
$$

where the summation $\sum_{i_{1}, \cdots, i_{m}} z_{i i_{1} \cdots i_{m}}$ is taken over all $d$-faces that $f_{i_{1}}, \ldots, f_{i_{m}}$ and $f_{i}$ sharing a same $(d-1)$-face. Moreover, $z_{i i_{1} \cdots i_{m}}$ is symmetric on its indices. The coordinate form of eigenvalue problem for down 1-Laplacian (3) is:

$$
\sum_{i_{1}, \cdots, i_{m}} z_{i i_{1} \cdots i_{m}} \in \mu(d+1) \operatorname{Sgn}\left(f_{i}\right), i=1,2, \ldots, q
$$

From the variational point of view, $\Delta_{1, d}^{u p}$ and $\Delta_{1, d}^{\text {down }}$ are respectively the subdifferential of the following convex functions

$$
I^{u p}(f)=\sum_{\bar{F} \in S_{d+1}(K)}\left|\sum_{F \prec \bar{F}} f_{F}\right|=\sum\left|f_{j_{1}}+\cdots+f_{j_{d+2}}\right|
$$

and

$$
I^{\text {down }}(f)=\sum_{E \in S_{d-1}(K)}\left|\sum_{F \succ E} f_{F}\right|=\sum\left|f_{j_{1}}+\cdots+f_{j_{m}}\right|
$$

i.e., $\Delta_{1, d}^{u p} f=\partial I^{u p}(f)$ and $\Delta_{1, d}^{\text {down }} f=\partial I^{\text {down }}(f)$. Indeed, the eigenvalue problem (1) for $\Delta_{1, d}^{u p}$ (resp. (3) for $\Delta_{1, d}^{\text {down }}$ ) could be derived from the variational problem of $I^{u p}(\cdot)$ (resp. 
$\left.I^{\text {down }}(\cdot)\right)$ on the piecewise linear manifold determined by $\|f\|^{\text {up }}=1$ (resp. $\|f\|^{\text {down }}=1$ ), where $\|f\|^{u p}=\sum_{i=1}^{q} \operatorname{deg}_{i}^{u p}\left|f_{i}\right|$ (resp. $\left.\|f\|^{\text {down }}=\sum_{i=1}^{q} \operatorname{deg}_{i}^{\text {down }}\left|f_{i}\right|\right)$.

It is known that critical points of $I^{u p}(\cdot) /\|\cdot\|^{\text {up }}\left(\right.$ resp., $I^{\text {down }}(\cdot) /\|\cdot\|^{\text {down }}$ ) must be eigenvectors of $\Delta_{1, d}^{u p}$ (resp., $\Delta_{1, d}^{\text {down }}$ ), and the minimal and maximal eigenvalues of $\Delta_{1, d}^{u p}$ (resp., $\left.\Delta_{1, d}^{\text {down }}\right)$ are respectively the minimum and maximum of $I^{\text {up }}(\cdot) /\|\cdot\|^{\text {up }}$ (resp., $I^{\text {down }}(\cdot) / \| \cdot$ $\left.\|^{\text {down }}\right)$. Moreover, the number of eigenvalues of $\Delta_{1, d}^{u p}$ (resp., $\left.\Delta_{1, d}^{\text {down }}\right)$ is finite. The proof is based on the standard and routine calculations similar to the related results in $[5,6,4]$ and thus we omit it.

\subsection{A glimpse of the spectrum of signless 1-Laplacian on complexes}

For a graph, we know that 0 is an eigenvalue of the signless 1-Laplacian if and only if the graph has a bipartite component. As a related analog on simplicial complex, we may take a look at the following result:

Proposition 1. $\Delta_{1, d}^{u p}$ has eigenvalue 0 if one of the following conditions holds.

(1) $\# S_{d}(K)>\# S_{d+1}(K)$;

(2) the up-degree of each $d$-face is less than or equal to $(d+2)$ and there is at least one $d$-face with the degree less than $(d+2)$;

(3) $S_{d}(K)$ is $(d+2)$ colorable, i.e., the colors of faces of each $(d+1)$-dim simplex are pairwise different.

Proof. (1) It is clear that 0 is an eigenvalue if and only if $I^{u p}(f)=0$ has a nonzero solution $f$, i.e., $f_{j_{1}}+\cdots+f_{j_{d+2}}=0, \forall \bar{F}_{j} \in S_{d+1}(K)$. Since the number of coordinate components of $f$ is $\# S_{d}(K)$ but the number of absolute terms of $I^{u p}$ is $\# S_{d+1}(K)$, and by $\# S_{d}(K)>\# S_{d+1}(K)$, the related linear system of equations has nonzero solution $f$. So there exists an eigenvector corresponding to 0.

(2) Since

$$
(d+2) \# S_{d+1}(K)=\sum_{i \in S_{d}(K)} \operatorname{deg}_{i}^{u p}<(d+2) \# S_{d}(K)
$$

implies $\# S_{d}(K)>\# S_{d+1}(K)$, it follows from (1) that (2) holds.

(3) Let $c: S_{d}(K) \rightarrow\{1, \ldots, d+2\}$ be a coloring map such that $\left\{c\left(f_{j_{1}}\right), \ldots, c\left(f_{j_{d+2}}\right)\right\}=$ $\{1, \ldots, d+2\}$ whenever $j_{1}, \ldots, j_{d+2}$ are $d$-dimensional faces of a $(d+1)$-dimensional simplex. Letting

$$
f_{i}= \begin{cases}1, & \text { if } c\left(f_{i}\right)=1 \\ -1, & \text { if } c\left(f_{i}\right)=2 \\ 0, & \text { otherwise }\end{cases}
$$

for $i=1, \ldots, q$, we have $f_{j_{1}}+\cdots+f_{j_{d+2}}=0$ whenever $j_{1}, \ldots, j_{d+2}$ are $d$-dimensional faces of a $(d+1)$-dimensional simplex. Thus, one can take $\mu=0$ and $z_{i j_{1} \cdots j_{d+1}}=0$ in (5). Consequently, 0 is an eigenvalue of $\Delta_{1, d}^{u p}$. 
Remark 2. Proposition 1 is only about the combinatorial properties of a simplicial complex, since our definition of signless 1-Laplacian doesn't involve the orientation of a simplicial complex. For example, let $K$ be determined by its facets $\{0,1,2,3\},\{1,2,3,4\}$ and $\{0,2,3,4\}$. Then $S_{2}(K)$ is 4 -colorable ${ }^{2}$, but $\{0,1,2,3\}$ and $\{1,2,3,4\}$ induce the different orientations on $\{1,2,3\}$.

Example 3. We show the detailed computation of spectrum of signless 1-Laplacian on the tetrahedron.

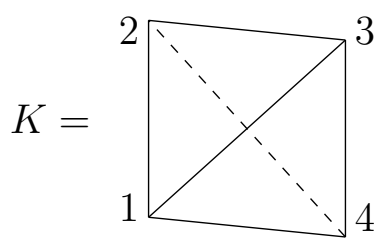

Consider the signless up 1-Laplacian for 2-faces $\{(123),(124),(134),(234)\}$. The eigenvalue problem is to solve the pair $(\mu, f) \in \mathbb{R} \times\left(\mathbb{R}^{n} \backslash\{0\}\right)$ such that

$$
\left\{\begin{array}{l}
\exists z_{1234} \in \operatorname{Sgn}\left(f_{123}+f_{124}+f_{134}+f_{234}\right), \\
\text { and } z_{123} \in \operatorname{Sgn}\left(f_{123}\right), z_{124} \in \operatorname{Sgn}\left(f_{124}\right), z_{134} \in \operatorname{Sgn}\left(f_{134}\right), z_{234} \in \operatorname{Sgn}\left(f_{234}\right) \text {, s.t. } \\
z_{1234}=\mu z_{123}=\mu z_{124}=\mu z_{134}=\mu z_{234} .
\end{array}\right.
$$

We first check $\mu=0$. In this situation, $z_{1234}=0$, which implies that $f_{123}+f_{124}+f_{134}+$ $f_{234}=0$. And $f=(1,-1,1,-1)$ is a solution.

Now we assume that $\mu \neq 0$. Since $f$ has a nonzero component, we can assume without loss of generality that $f_{123}>0$, which implies that $z_{123}=1$. Note that $\mu=\mu z_{123}=\mu z_{124}$, which follows that $z_{124}=1$ and thus $f_{124} \geqslant 0$. Similarly, we have $f_{134} \geqslant 0$ and $f_{234} \geqslant 0$. So, $f_{123}+f_{124}+f_{134}+f_{234}>0$ and then $z_{1234}=1$. Consequently, $1=z_{1234}=\mu z_{123}=\mu$. And $f=(1,0,0,0)$ is a solution. Therefore, $\mu=1$ is the eigenvalue.

In summary, the spectrum of signless 1-Laplacian for 2 -faces of $K$ is $\{0,1\}$.

Remark 4. In fact, the above example could be generalized to $\Delta_{1, d-1}^{u p}$ for $d$-dimensional simplex. That is, the spectrum of $\Delta_{1, d-1}^{u p}$ for $d$-dimensional simplex is $\{0,1\}$.

\section{Courant nodal domain theorem}

In this section, we develop Courant nodal domain theorem for 1-Laplacian on complexes.

Similar to the reason (see [4] Page 8), we should modify the definition of nodal domain as follows.

Definition 3. A set of $d$-simplexes is up-connected (resp., down-connected), if for any $\sigma$ and $\sigma^{\prime}$ in such set, there exists a sequence of $\left\{\sigma_{i}\right\}_{i=0}^{i=m}$ such that $\sigma=\sigma_{0} \stackrel{\text { up }}{\sim} \sigma_{1} \stackrel{\text { up }}{\sim} \ldots \stackrel{\text { up }}{\sim} \sigma_{m}=\sigma^{\prime}$ (resp., $\sigma=\sigma_{0} \stackrel{\text { down }}{\sim} \sigma_{1} \stackrel{\text { down }}{\sim} \ldots \stackrel{\text { down }}{\sim} \sigma_{m}=\sigma^{\prime}$ ).

\footnotetext{
${ }^{2}$ Indeed, in this example, all the conditions (1),(2),(3) in Proposition 1 hold.
} 
Definition 4. The (up-/down-) nodal domains of an eigenvector $f=\left(f_{1}, f_{2}, \ldots, f_{q}\right)$ of $\Delta_{1}$ are defined to be maximal (up-/down-) connected components of the support set $D(f):=\left\{i: f_{i} \neq 0\right\}$.

Proposition 2. Suppose $(\mu, f)$ is an eigenpair of the signless (up-/down-) 1-Laplacian and $D_{1}, \ldots, D_{k}$ are (up-/down-) nodal domains of $f$. Let $f^{i}$ be defined as

$$
f_{j}^{i}= \begin{cases}\frac{f_{j}}{\sum_{j \in D_{i}(f)} \operatorname{deg}_{j}\left|f_{j}\right|}, & \text { if } j \in D_{i}(f) \\ 0, & \text { if } j \notin D_{i}(f)\end{cases}
$$

for $j=1,2, \ldots, q$. Then $\left(\mu, f^{i}\right)$ is an eigenpair, $i=1,2, \ldots, k$.

Proof. It can be directly verified that $i \stackrel{\text { up }}{\sim} j$ (resp. $i \stackrel{\text { down }}{\sim} j$ ) if and only if $f_{i}$ and $f_{j}$ appear in a same term $|\cdot|$ in the summation form of $I^{u p}(\cdot)$ (resp. $\left.I^{\text {down }}(\cdot)\right)$. Hence, we derive that $\operatorname{Sgn}\left(f_{j}^{i}\right) \supset \operatorname{Sgn}\left(f_{j}\right)$ and $\operatorname{Sgn}\left(f_{j}^{i}+f_{j_{1}}^{i}+\cdots+f_{j_{m}}^{i}\right) \supset \operatorname{Sgn}\left(f_{j}+f_{j_{1}}+\cdots+f_{j_{m}}\right), j^{\prime} \sim j$, $j=1,2, \ldots, n, i=1,2, \ldots, k$. Then, using the coordinate form of 1-Laplacian eigenvalue problems (5) and (6), we complete the proof.

Fixing the dimension $d$ and $n:=\# S_{d}(K)$, denote $I(\cdot)=I^{u p}(\cdot)\left(\operatorname{resp} . I^{\text {down }}(\cdot)\right)$ and $\|\cdot\|=\|\cdot\|^{\text {up }}$ (resp. $\|\cdot\|^{\text {down}}$ ). We apply the Liusternik-Schnirelmann theory to $\Delta_{1}$. Note that $I(f)$ is even, and $X=\{f:\|f\|=1\}$ is symmetric. For a symmetric set $T \subset X$, i.e., $-T=T$, the Krasnoselski genus (see $[8,25]$ ) of $T$ is defined to be

$$
\gamma(T)= \begin{cases}0, & \text { if } T=\varnothing, \\ \min \left\{k \in \mathbb{Z}: \exists \text { odd continuous } h: T \rightarrow \mathbb{S}^{k-1}\right\}, & \text { otherwise }\end{cases}
$$

Obviously, the genus is a topological invariant. Let us define

$$
c_{k}=\inf _{\gamma(T) \geqslant k} \max _{f \in T \subset X} I(f)=\inf _{\gamma(T) \geqslant k} \max _{f \in T \subset \mathbb{R}^{n} \backslash\{0\}} \frac{I(f)}{\|f\|}, \quad k=1,2, \ldots n .
$$

By the same way as already used in [5], it can be proved that these $c_{k}$ are critical values of $I(f)$. One has

$$
c_{1} \leqslant c_{2} \leqslant \cdots \leqslant c_{n}
$$

and if $0 \leqslant \cdots \leqslant c_{k-1}<c_{k}=\cdots=c_{k+r-1}<c_{k+r} \leqslant \cdots \leqslant 1$, the multiplicity of $c_{k}$ is defined to be $r$. The Courant nodal domain theorem for the signless 1-Laplacian reads

Theorem 5. Let $c_{k}$ be an eigenvalue with multiplicity $r$ and let $f^{k}$ be an eigenvector associated with $c_{k}$. Then

$$
1 \leqslant N\left(f^{k}\right) \leqslant k+r-1
$$

where $N\left(f^{k}\right)$ is the number of nodal domains of $f^{k}$.

Proof. Assume there are $n d$-faces in the complex. Suppose the contrary, that there exists an eigenvector $f^{k}=\left(f_{1}, f_{2}, \ldots, f_{n}\right)$ corresponding to the variational eigenvalue $c_{k}$ with 
multiplicity $r$ such that $N\left(f^{k}\right) \geqslant k+r$. Let $D_{1}\left(f^{k}\right), \ldots, D_{k+r}\left(f^{k}\right)$ be the nodal domains of $f^{k}$. Let $g^{i}=\left(g_{1}^{i}, g_{2}^{i}, \ldots, g_{n}^{i}\right)$, where

$$
g_{j}^{i}= \begin{cases}\frac{f_{j}}{\sum_{j \in D_{i}(f)} \operatorname{deg}_{j}\left|f_{j}\right|}, & \text { if } j \in D_{i}(f), \\ 0, & \text { if } j \notin D_{i}(f),\end{cases}
$$

for $i=1,2, \ldots, k+r, j=1,2, \ldots, n$. By the construction of $g^{i}, i=1,2, \ldots, k+r$, we have:

(1) The nodal domain of $g^{i}$ is the $i$-th nodal domain of $f^{k}$, i.e., $D\left(g^{i}\right)=D_{i}\left(f^{k}\right)$;

(2) $D\left(g^{i}\right) \cap D\left(g^{j}\right)=\varnothing, i \neq j$;

(3) By Proposition $2, g^{1}, \ldots, g^{k+r}$ are all eigenvectors with the same eigenvalue $c_{k}$.

Now, for any $f \in \operatorname{span}\left(g^{1}, \cdots, g^{k+r}\right) \cap X$, there exist $a_{1}, \ldots, a_{k+r}$ such that $f=$ $\sum_{i=1}^{k+r} a_{i} g^{i} \in X$. And for any $l \in\{1,2, \ldots, n\}$, there exists a unique $j$ such that $f_{l}=a_{j} g_{l}^{j}$. Hence, $\left|f_{l}\right|=\sum_{j=1}^{k+r}\left|a_{j}\right|\left|g_{l}^{j}\right|$. Since $f \in X, g^{j} \in X, j=1, \ldots, k+r$, we have

$$
1=\sum_{l=1}^{n} \operatorname{deg}_{l}\left|f_{l}\right|=\sum_{l=1}^{n} \operatorname{deg}_{l} \sum_{j=1}^{k+r}\left|a_{j}\right|\left|g_{l}^{j}\right|=\sum_{j=1}^{k+r}\left|a_{j}\right| \sum_{l=1}^{n} \operatorname{deg}_{l}\left|g_{l}^{j}\right|=\sum_{j=1}^{k+r}\left|a_{j}\right| .
$$

Note that $I(\cdot)$ is convex and even. Therefore, we have

$$
\begin{aligned}
I(f) & =I\left(\sum_{i=1}^{k+r} a_{i} g^{i}\right)=I\left(\sum_{i=1}^{k+r}\left|a_{i}\right| \operatorname{sgn}\left(a_{i}\right) g^{i}\right) \\
& \leqslant \sum_{i=1}^{k+r}\left|a_{i}\right| I\left(\operatorname{sgn}\left(a_{i}\right) g^{i}\right) \leqslant \sum_{i=1}^{k+r}\left|a_{i}\right| I\left(g^{i}\right) \\
& \leqslant \max _{i=1,2, \ldots, k+r} I\left(g^{i}\right) .
\end{aligned}
$$

Note that $g^{1}, \ldots, g^{k+r}$ are non-zero orthogonal vectors, so $\operatorname{span}\left(g^{1}, \ldots, g^{k+r}\right)$ is a $k+r$ dimensional linear space. It follows that $\operatorname{span}\left(g^{1}, \ldots, g^{k+r}\right) \cap X$ is a symmetric set which is homeomorphous to $\mathbb{S}^{k+r-1}$. Obviously, $\gamma\left(\operatorname{span}\left(g^{1}, \ldots, g^{k+r}\right) \cap X\right)=k+r$. Therefore, we derive that

$$
\begin{aligned}
c_{k+r} & =\inf _{\gamma(A) \geqslant k+r} \sup _{f \in A} I(f) \\
& \leqslant \sup _{f \in \operatorname{span}\left(g^{1}, \ldots, g^{k+r}\right) \cap X} I(f) \\
& =\max _{i=1, \ldots, k+r} I\left(g^{i}\right) \\
& =c_{k},
\end{aligned}
$$

which contradicts with the fact $c_{k}<c_{k+r}$. The proof is completed. 


\section{On some combinatorial parameters of complexes}

In this section, we concentrate on the relationships between the eigenvalues of signless 1Laplacian and other combinatorial parameters, such as chromatic number, independence number and clique covering number.

\subsection{Independence number and chromatic number for vertices}

Firstly, we recall the concepts of independence number and chromatic number of a hypergraph. The definition of chromatic number of hypergraphs generalize chromatic number of graphs in various ways, see for example [19]. The chromatic number for $r$-unifrorm hypergraphs is defined as

$$
\chi_{s}=\min \left\{k: \exists k \text {-partition }\left(V_{1}, \ldots, V_{k}\right) \text { such that }\left|V_{i} \cap E\right| \leqslant s, \forall \text { edge } E, \forall i\right\},
$$

while the independence number of a hypergraph is defined by

$$
\alpha_{s}=\max \{|A|:|A \cap E| \leqslant s, \forall \text { edge } E\}
$$

for $1 \leqslant s \leqslant r-1$ (see [19]). Note that a simplicial complex can be regarded as a hypergraph. Thus, the definition of independence number and chromatic number for simplicial complexes can be defined as follows:

Definition 5 (Independence number). For a simplicial complex $K=(V, S)$, the independence number is

$$
\alpha_{s}=\max \{|A|:|A \cap F| \leqslant s, \forall \text { face } F\} .
$$

Definition 6 (Chromatic number). For a simplicial complex $K=(V, S)$, the chromatic number is

$$
\chi_{s}=\min \left\{k: \exists k \text {-partition }\left(V_{1}, \ldots, V_{k}\right) \text { such that }\left|V_{i} \cap F\right| \leqslant s, \forall \text { face } F, \forall i\right\} .
$$

In [11], the independence number and chromatic number of a simplicial complex are respectively defined by

$$
\alpha=\max \{|A|: A \not \supset F, \forall \text { maximal face } F\}
$$

and

$$
\chi=\min \left\{k: \exists k \text {-partition }\left(V_{1}, \ldots, V_{k}\right) \text { such that } V_{i} \not \supset F, \forall \text { maximal face } F, \forall i\right\}
$$

However, in the proof of main theorems in [11], the author essentially deals with $\alpha_{d}$ and $\chi_{d}$, where $d$ is the dimension of complex. That is, the results still hold if we replace ' $\alpha$ ' and ' $\chi$ ' by ' $\alpha_{d}$ ' and ' $\chi_{d}$ ' in those theorems. In this subsection, we will concentrate on $\chi_{s}$ and $\alpha_{s}$ and study their relations with eigenvalues of 1-Laplacian. An elementary result for the relations of these concepts is:

Proposition 3. For simplicial complex $K=(V, S)$, we have: 
(1) $\chi_{s} \alpha_{s} \geqslant|V|, \chi_{s} \leqslant\left\lceil\chi_{1} / s\right\rceil$, for all $s$. Here $\lceil x\rceil$ is the ceiling function on $x$, i.e., the minimum integer that does not less than $x$. Moreover, $\chi_{s} \leqslant\left\lceil\chi_{t} /\lfloor s / t\rfloor\right\rceil$, where $\lfloor x\rfloor$ is the Gauss function on $x$, i.e., the maximum integer that does not exceed $x$.

(2) $\chi \alpha \geqslant|V|, \chi_{d} \leqslant \chi \leqslant\left\lceil\chi_{1} / d\right\rceil, \alpha \leqslant \alpha_{d}$, where $d$ is the dimension of the complex. For a pure (i.e., homogeneous) complex, $\alpha=\alpha_{d}$.

(3) The above definitions for independence number $\alpha_{s}$ and chromatic number $\chi_{s}$ are respectively equivalent to

$$
\alpha_{s}^{\prime}=\max \{|A|:|A \cap F| \leqslant s, \forall s \text {-face } F\}
$$

and

$$
\chi_{s}^{\prime}=\min \left\{k: \exists k \text {-partition }\left(V_{1}, \ldots, V_{k}\right) \text { such that }\left|V_{i} \cap F\right| \leqslant s, \forall \text { s-face } F, \forall i\right\} \text {. }
$$

Proof.

(1) It is evident that $\chi_{s} \alpha_{s} \geqslant|V|$ by the definitions. To prove $\chi_{s} \leqslant \chi_{1} / s$, let $\left(V_{1}, \ldots, V_{\chi_{1}}\right)$ be a partition of $V$ such that $\left|V_{i} \cap F\right| \leqslant 1$, for any face $F$. Let $U_{i}=V_{(i-1) s+1} \cup \cdots \cup V_{i s}$ for $i=1, \ldots, m-1$, and $U_{m}=V_{(m-1) s+1} \cup \cdots \cup V_{\chi_{1}}$, where $m=\left\lceil\chi_{1} / s\right\rceil$. Then $\left|U_{i} \cap F\right| \leqslant \sum_{j=1}^{s}\left|V_{(i-1) s+j} \cap F\right| \leqslant s, i=1, \ldots, m$. Therefore, $\chi_{s} \leqslant m$.

The rest of the proof is similar with the above, but we shall provide the details for reader's convenience. Let $\left(V_{1}, \ldots, V_{\chi t}\right)$ be a partition of $V$ such that $\left|V_{i} \cap F\right| \leqslant t, \forall F$. Let $U_{i}=V_{(i-1) s^{\prime}+1} \cup \cdots \cup V_{i s^{\prime}}$ for $i=1, \ldots, m-1$, and $U_{m}=V_{(m-1) s^{\prime}+1} \cup \cdots \cup V_{\chi_{t}}$, where $m=\left\lceil\chi_{t} / s^{\prime}\right\rceil$ and $s^{\prime}=\lfloor s / t\rfloor$. Then

$$
\left|U_{i} \cap F\right| \leqslant \sum_{j=1}^{s^{\prime}}\left|V_{(i-1) s^{\prime}+j} \cap F\right| \leqslant s^{\prime} t=\lfloor s / t\rfloor t \leqslant s,
$$

for $i=1, \ldots, m$. Therefore, $\chi_{s} \leqslant m$.

(2) Note that $\chi \alpha \geqslant|V|$ and $\chi \leqslant\left\lceil\chi_{1} / d\right\rceil$ have been proved in [11]. Now we prove $\alpha \leqslant \alpha_{d}$. Since the dimension of $K$ is $d$, the maximal face $F$ has at most $d+1$ elements. So $F \not \subset A$ implies $|F \cap A| \leqslant|F|-1 \leqslant d$, for any maximal face $F$. Thus, $|F \cap A| \leqslant d$ holds for any face $F$. This deduces that

$$
\{|A|: A \not \supset F, \forall \text { maximal face } F\} \subset\{|A|:|A \cap F| \leqslant d, \forall \text { face } F\} .
$$

Therefore, $\alpha \leqslant \alpha_{d}$. Similarly, $\chi \geqslant \chi_{d}$.

A maximal face (i.e., facet) is not necessary to be a $d$-face. But if the complex is homogeneous (or pure), then facets must coincide with $d$-faces. So, it is easy to check that $\alpha=\alpha_{d}$. 
(3) It is easy to check that $\alpha_{s}^{\prime} \geqslant \alpha_{s}$. Next we will show the reverse inequality. Indeed, it is enough to show that for any set $A$ with $|A \cap F| \leqslant s$ for all $s$-faces $F$, we have $|A \cap F| \leqslant s$ for any face whose dimension is larger than $s$. For the contrary, there is some $(s+k)$-face $F^{\prime}$ satisfying $\left|A \cap F^{\prime}\right| \geqslant s+1$. Then there is one $s$-face whose vertices are in $A$, which is a contradiction. The proof for $\chi_{s}^{\prime}=\chi_{s}$ is similar.

Remark 6 . There exists a complex $K$ such that $\alpha<\alpha_{d}$, where $d=\operatorname{dim}(K)$. Indeed, let $V=\{1,2,3,4,5\}$ and let

$K=\{\{1\},\{2\},\{3\},\{4\},\{5\},\{1,2\},\{1,3\},\{2,3\},\{1,2,3\},\{1,4\},\{2,5\}\}$. Then $K$ is a complex of dimension $d=2$. It can be verified that $\alpha=3(\{3,4,5\}$ is an independent set) and $\alpha_{d}=4$ with a corresponding independent set $\{1,2,4,5\}$.

Theorem 7. Let $K=(V, S)$ be a nonempty d-dimensional complex. Then

$$
\alpha \leqslant \alpha_{d} \leqslant \min \left\{|V|\left(1-\frac{\mu_{d-1}}{2 M_{0}}\right),|V| \frac{M_{0}}{M_{0}+\mu_{d-1}},|V| \frac{M_{0}-\mu_{0}}{M_{0}+m_{0}}\right\}
$$

and hence

$$
\chi \geqslant \chi_{d} \geqslant \max \left\{\frac{2 M_{0}}{2 M_{0}-\mu_{d-1}}, 1+\frac{\mu_{d-1}}{M_{0}}, \frac{M_{0}+m_{0}}{M_{0}-\mu_{0}}\right\}
$$

where $M_{0}$ and $m_{0}$ are the largest and smallest degree of vertices in $K, \mu_{d-1}$ is the minimum unnormalized eigenvalue of $\Delta_{1, d-1}^{u p}$, and similarly $\mu_{0}$ is the minimum unnormalized eigenvalue of $\Delta_{1,0}^{u p}$.

Proof. Let $A \subset V$ be the largest independent set with $|A|=\alpha_{d}$, and for some fixed constants $a, b \in \mathbb{R}$, let

$$
f_{i}= \begin{cases}a, & i \in A \\ b, & i \in A^{c}:=V \backslash A\end{cases}
$$

Then

$$
\begin{aligned}
\sum_{i \sim j}\left|f_{i}+f_{j}\right| & =\sum_{i \sim j, i \in A, j \in A^{c}}|a+b|+\sum_{i \sim j \text { in } A} 2|a|+\sum_{i \sim j \text { in } A^{c}} 2|b| \\
& =|a+b| \cdot\left|E\left(A, A^{c}\right)\right|+|b|\left(\operatorname{vol}\left(A^{c}\right)-\left|E\left(A, A^{c}\right)\right|\right)
\end{aligned}
$$

and

$$
\sum_{i \in V}\left|f_{i}\right|=|a||A|+|b|\left|A^{c}\right|
$$

Thus,

$$
\max _{i \in V} \operatorname{deg}_{i}^{u p}=\max _{\vec{g} \neq \overrightarrow{0}} \frac{\sum_{i \sim j}\left|g_{i}+g_{j}\right|}{\sum_{i \in V}\left|g_{i}\right|} \geqslant \frac{\sum_{i \sim j}\left|f_{i}+f_{j}\right|}{\sum_{i \in V}\left|f_{i}\right|} \geqslant \frac{|a+b| \cdot\left|E\left(A, A^{c}\right)\right|}{|a||A|+|b|\left|A^{c}\right|} .
$$

Taking $a=\left|A^{c}\right|$ and $b=|A|$, we get

$$
\max _{i \in V} \operatorname{deg}_{i}^{u p} \geqslant\left|E\left(A, A^{c}\right)\right| \frac{|V|}{2 \alpha_{d}\left(|V|-\alpha_{d}\right)} .
$$


Let $M_{i}$ and $m_{i}$ be the maximum and minimum number of $(i+1)$-faces that contains an $i$-face, respectively, $i \in\{0,1, \ldots, d-1\}$.

Since $A$ is independent, for each $(d-1)$-face $F \subset A$, the number of $d$-faces that contains $F$ and meets $A^{c}$ is larger than or equals to $m_{d-1}$. And it can be proved that $m_{0}>m_{1}>\cdots>m_{d-1}$.

Claim: for any $i$-face $F \subset A$ with $i \in\{0, \ldots, d-1\}$, the number of $(i+1)$-faces that contains $F$ and meets $A^{c}$ is larger than or equal to $m_{d-1}$.

We prove the above claim by induction on $i$ from $(d-1)$ to 0 .

For $i=d-1$, the claim holds according to the definition of $m_{d-1}$ and the independence of $A$.

Suppose the claim holds for $i$. Then for the case of $(i-1)$, for any $(i-1)$-face $F$, there are two subcases:

1. $F$ contains in an $i$-face $F^{\prime}$ with all its vertices in $A$.

In this case, using the inductive hypothesis for $F^{\prime}$, there exist $v_{1}, \ldots, v_{m_{d-1}} \in A^{c}$ such that $F^{\prime} \cup\left\{v_{1}\right\}, \ldots, F^{\prime} \cup\left\{v_{m_{d-1}}\right\}$ are $(i+1)$-faces. In consequence, $F \cup\left\{v_{1}\right\}$, $\ldots, F \cup\left\{v_{m_{d-1}}\right\}$ are $i$-faces, which means that the claim holds for such $F$.

2. There is no $i$-face with all its vertices in $A$ that contains $F$.

In this case, all $i$-faces that contains $F$ must meet $A^{c}$, and the number of $i$-faces containing $F$ is at least $m_{i-1} \geqslant m_{d-1}$. Thus the claim holds.

In particular, for $i=0$, it means that $\left|E\left(\{i\}, A^{c}\right)\right| \geqslant m_{d-1}$ for any $i \in A$. Thus,

$$
\left|E\left(A, A^{c}\right)\right|=\sum_{i \in A}\left|E\left(\{i\}, A^{c}\right)\right| \geqslant|A| m_{d-1}=\alpha_{d} m_{d-1} .
$$

Taking $\vec{g}=\vec{e}_{F_{0}}$, where $F_{0}$ is a $(d-1)$-face such that $\mid\left\{i \in F_{0}^{c}: F_{0} \cup\{i\}\right.$ is $d$-face $\} \mid=$ $m_{d-1}$. Then $m_{d-1}=I^{u p}(\vec{g}) /\|\vec{g}\|_{1} \geqslant \mu_{d-1}$ and hence,

$$
\left|E\left(A, A^{c}\right)\right| \geqslant \alpha_{d} \mu_{d-1} .
$$

Combining (8) and (9), we have

$$
M_{0}=\max _{i \in V} \operatorname{deg}_{i}^{u p} \geqslant \mu_{d-1} \frac{|V|}{2\left(|V|-\alpha_{d}\right)},
$$

which derives our desired inequality.

It is also clear that $\alpha_{d} m_{0} \leqslant\left|E\left(A, A^{c}\right)\right| \leqslant \alpha_{d} M_{0}$ and $\operatorname{vol}\left(A^{c}\right) \leqslant\left(|V|-\alpha_{d}\right) M_{0}$.

Taking $a=-1$ and $b=1$, we obtain

$\mu_{0}=\inf _{\vec{g} \neq \overrightarrow{0}} \frac{\sum_{i \sim j}\left|g_{i}+g_{j}\right|}{\sum_{i \in V}\left|g_{i}\right|} \leqslant \frac{\sum_{i \sim j}\left|f_{i}+f_{j}\right|}{\sum_{i \in V}\left|f_{i}\right|}=\frac{\operatorname{vol}\left(A^{c}\right)-\left|E\left(A, A^{c}\right)\right|}{|V|} \leqslant \frac{\left(|V|-\alpha_{d}\right) M_{0}-\alpha_{d} m_{0}}{|V|}$

which implies $\alpha_{d} \leqslant|V| \frac{M_{0}-\mu_{0}}{M_{0}+m_{0}}$. 
Note that

$$
\max _{a b \neq 0} \frac{|a+b|}{|a||A|+|b|\left|A^{c}\right|}=\max \left\{\frac{1}{|A|}, \frac{1}{\left|A^{c}\right|}\right\}=\max \left\{\frac{1}{\alpha_{d}}, \frac{1}{|V|-\alpha_{d}}\right\} .
$$

This implies

$$
\max _{i \in V} \operatorname{deg}_{i}^{u p} \geqslant\left|E\left(A, A^{c}\right)\right| \max \left\{\frac{1}{\alpha_{d}}, \frac{1}{|V|-\alpha_{d}}\right\} .
$$

Combining (10) and (9), we have

$$
M_{0}=\max _{i \in V} \operatorname{deg}_{i}^{u p} \geqslant \mu_{d-1} \max \left\{1, \frac{\alpha_{d}}{|V|-\alpha_{d}}\right\},
$$

which derives $\alpha_{d} \leqslant \frac{M_{0}}{M_{0}+\mu_{d-1}}|V|$.

Remark 8. Here the proof of Theorem 7 is inspired by the technique of the proof of Theorem 2 in [11].

We can also give a lower bound for the independence number by means of the proof of Theorem 7. Since $A$ is the maximal independent set, for any $j \in A^{c}, A \cup\{j\}$ is not independent, i.e., there is a $d$-face in $A \cup\{j\}$ containing $j$ as its vertex, which implies that $|E(A,\{j\})| \geqslant d$. Thus,

$$
\left|E\left(A, A^{c}\right)\right|=\sum_{j \in A^{c}}|E(A,\{j\})| \geqslant\left|A^{c}\right| d=\left(|V|-\alpha_{d}\right) d .
$$

Combining (10) and (11), we have

$$
\alpha_{d} \geqslant|V| \frac{d}{M_{0}+d} .
$$

Combining (8) and (11), we have

$$
\alpha_{d} \geqslant|V| \frac{d}{2 M_{0}} .
$$

Remark 9. In the one-dimensional case, i.e., the graph case, we note Hoffman's result $\alpha \leqslant|V| \frac{\lambda_{\max }-m_{0}}{\lambda_{\max }}$, where $\lambda_{\max }$ is the largest eigenvalue of the Laplacian, $m_{0}$ is the minimal degree. For a 5 -order cyclical graph $G=(V, E)$ with $V=\{1,2,3,4,5\}$ and $E=\{\{1,2\},\{2,3\},\{3,4\},\{4,5\},\{5,1\}\}, \lambda_{\max }(G)=2-2 \cos \frac{4}{5} \pi \approx 3.618$ and thus Hoffman's upper bound $\approx 5 \times \frac{3.618-2}{3.618}=2.23$. Note that the upper bound in Theorem 7 is $5 \times \frac{2-\frac{2}{5}}{4}=2$, which is better than Hoffman's upper bound.

Since Theorem 1.2 in [11] coincides with Hoffman's bound when the simplicial complex is 1-dimensional, it is clear that Theorem 7 could provide better bounds on some special cases. That is, Theorem 7 can be comparable to some kinds of Hoffman's bound like Theorem 1.2 in [11].

So far, we focused on vertices of simplicial complexes, i.e. 0-faces. In fact, we can also define independence number and chromatic number on $i$-faces of simplicial complexes. In the following subsection, we will introduce corresponding definitions and study the relationships between eigenvalues of 1-Laplacian and them. 


\subsection{Chromatic number and dual Cheeger constant for $d$-faces}

Definition 7 (chromatic number). A $d$-face coloring of a simplicial complex assigns a color to each $d$-face so that no two faces that contain in the same $(d+1)$-face have the same color. The smallest number of colors needed is called its chromatic (or coloring) number.

In this subsection, we will use $\chi$ to denote the chromatic number of $S_{d}$ (the set of $d$-faces). One can easily see that the chromatic number of all $d$-faces is at least $d+2$.

Definition 8 (dual Cheeger constant). The dual Cheeger constant on the set $S_{d}$ consisting of $d$-faces is defined as

$$
h^{u p *}=\max _{A \cap B=\varnothing \neq A \cup B \subset S_{d}} \frac{2\left|E_{S_{d}}(A, B)\right|}{\operatorname{vol}(A)+\operatorname{vol}(B)}
$$

where $E_{S_{d}}(A, B)$ is the collection of all $(d+1)$-simplices that has some $d$-face in $A$ and also has some $d$-face in $B$. Hereafter, $\operatorname{vol}(S):=\sum_{i \in S} \operatorname{deg}^{u p}(i)$ is the volume of $S$, for any $S \subset S_{d}$

Theorem 10. Let $\|f\|=\sum_{i \in S_{d}} \operatorname{deg}^{u p}(i)\left|f_{i}\right|$ for any $f \in \mathbb{R}^{S_{d}}$. Then

$$
\mu_{1}^{u p} \leqslant 1-\frac{2(d+1)}{d+\chi} \quad \text { and } \quad \chi \geqslant \frac{2(d+1)}{h^{u p *}}-d .
$$

where $\mu_{1}^{u p}=\min _{f \neq 0} \frac{I^{u p}(f)}{\|f\|}$. Furthermore, these bounds are sharp.

Proof of Theorem 10. Let $S_{d}^{1}, \ldots, S_{d}^{\chi}$ be the color classes of $S_{d}$. Given an integer $k \in$ $\{1,2, \ldots, \chi\}$, we define the vector $f$ by

$$
f_{i}= \begin{cases}a, & \text { if } i \in S_{d}^{k} \\ b, & \text { if } i \notin S_{d}^{k}\end{cases}
$$

We have

$$
\mu_{1}^{u p}\|f\| \leqslant I^{u p}(f)
$$

It is evident that

$$
\|f\|=|a| \operatorname{vol}\left(S_{d}^{k}\right)+|b| \operatorname{vol}\left(S_{d} \backslash S_{d}^{k}\right)=(|a|-|b|) \operatorname{vol}\left(S_{d}^{k}\right)+|b| \operatorname{vol}\left(S_{d}\right),
$$

and

$$
\begin{aligned}
I^{u p}(f) & =|a+(d+1) b| e_{0, d}\left(S_{d}^{k}, S_{d} \backslash S_{d}^{k}\right)+(d+2)|b| e_{d+1}\left(S_{d} \backslash S_{d}^{k}\right) \\
& =(|a+(d+1) b|-(d+2)|b|) e_{0, d}\left(S_{d}^{k}, S_{d} \backslash S_{d}^{k}\right)+(d+2)|b| e_{d+1}\left(S_{d}\right),
\end{aligned}
$$

where $e_{0, d}\left(S_{d}^{k}, S_{d} \backslash S_{d}^{k}\right)$ counts the number of $(d+1)$-simplexes with one $d$-face in $S_{d}^{k}$ and others in $S_{d} \backslash S_{d}^{k}$, and $e_{d+1}\left(S_{d} \backslash S_{d}^{k}\right)$ (resp. $\left.e_{d+1}\left(S_{d}\right)\right)$ is the number of $(d+1)$-simplexes with $d$-faces in $S_{d} \backslash S_{d}^{k}$ (resp. $S_{d}$ ). 
In summary, for every $k=1, \ldots, \chi$, we have

$\mu_{1}^{u p}\left((|a|-|b|) \operatorname{vol}\left(S_{d}^{k}\right)+|b| \operatorname{vol}\left(S_{d}\right)\right) \leqslant(|a+(d+1) b|-(d+2)|b|) e_{0, d}\left(S_{d}^{k}, S_{d} \backslash S_{d}^{k}\right)+(d+2)|b| e_{d+1}\left(S_{d}\right)$.

Summing these inequalities for $k=1,2, \cdots, \chi$, we obtain $\mu_{1}^{u p} \sum_{k=1}^{\chi}\left((|a|-|b|) \operatorname{vol}\left(S_{d}^{k}\right)+\right.$ $\left.|b| \operatorname{vol}\left(S_{d}\right)\right) \leqslant \sum_{k=1}^{\chi}\left((|a+(d+1) b|-(d+2)|b|) e_{0, d}\left(S_{d}^{k}, S_{d} \backslash S_{d}^{k}\right)+(d+2)|b| e_{d+1}\left(S_{d}\right)\right)$. Elementary computation gives

$\mu_{1}^{u p}\left((|a|-|b|) \operatorname{vol}\left(S_{d}\right)+|b| \operatorname{vol}\left(S_{d}\right) \chi\right) \leqslant(|a+(d+1) b|-(d+2)|b|)(d+2) e_{d+1}\left(S_{d}\right)+(d+2)|b| e_{d+1}\left(S_{d}\right) \chi$.

Now we get

$$
\begin{aligned}
\mu_{1}^{u p} & \leqslant \frac{(d+2) e_{d+1}\left(S_{d}\right)}{\operatorname{vol}\left(S_{d}\right)} \frac{|a+(d+1) b|-(d+2)|b|+|b| \chi}{|a|-|b|+|b| \chi} \\
& =\frac{(d+2) e_{d+1}\left(S_{d}\right)}{\operatorname{vol}\left(S_{d}\right)}\left(1-\frac{|a|+(d+1)|b|-|a+(d+1) b|}{|a|+(\chi-1)|b|}\right) .
\end{aligned}
$$

It is easy to see that

$$
\max _{(a, b) \neq(0,0)} \frac{|a|+(d+1)|b|-|a+(d+1) b|}{|a|+(\chi-1)|b|}=\max _{t \leqslant 0} \frac{|t|+(d+1)-|t+(d+1)|}{|t|+(\chi-1)}=\frac{2(d+1)}{d+\chi}
$$

where the maximum arrives at $t=-d-1$, and this implies the desired inequality

$$
\mu_{1}^{u p} \leqslant \frac{(d+2) e_{d+1}\left(S_{d}\right)}{\operatorname{vol}\left(S_{d}\right)}\left(1-\frac{2(d+1)}{d+\chi}\right)
$$

By the elementary fact $(d+2) e_{d+1}\left(S_{d}\right)=\operatorname{vol}\left(S_{d}\right)$, we have

$$
\mu_{1}^{u p} \leqslant 1-\frac{2(d+1)}{d+\chi}
$$

Using the equality $\mu_{1}^{u p}+h^{u p *}=1$, we get $\frac{2(d+1)}{d+\chi} \leqslant h^{u p *}$ and thus $\chi \geqslant \frac{2(d+1)}{h^{u p *}}-d$.

Finally, we prove that the bounds are sharp. In fact, if $K$ is a $(d+1)$-simplex, then these equalities hold.

Remark 11. It is worth noting that Proposition 1 (3) is a special case of Theorem 10 by taking $\chi=d+2$.

Corollary 1. Let $\chi$ be the classical chromatic number of a graph, then

$$
\mu_{1}^{u p} \leqslant 1-\frac{2}{\chi} \text { and } \frac{2}{h^{u p *}} \leqslant \chi .
$$

\footnotetext{
${ }^{3}$ This relation can be proved in the same way as shown in [4].
} 
Remark 12. For the normalized signless Laplacian on a graph [18], we similarly have $\lambda_{1}^{u p} \leqslant 1-\frac{1}{\chi-1}$. And combining with Corollary 1, we obtain

$$
\chi \geqslant \max \left\{\frac{2}{1-\mu_{1}^{u p}}, \frac{1}{1-\lambda_{1}^{u p}}+1\right\} .
$$

Interestingly, by dual Cheeger inequality, there is an inequality

$$
\frac{\lambda_{1}^{u p}}{2} \leqslant \mu_{1}^{u p} \leqslant \sqrt{\lambda_{1}^{u p}\left(2-\lambda_{1}^{u p}\right)}
$$

where $\lambda_{1}^{u p}$ is the smallest eigenvalue of signless Lapalacian and $\mu_{1}^{u p}$ is the minimal eigenvalue of signless 1-Lapalacian.

Hoffman [14] has the following estimate $\chi \geqslant 1-\frac{\lambda_{\max }(A)}{\lambda_{\min }(A)}$ of the chromatic number involving adjacent matrix $A$ of a graph. Some update results can be found in [26]. Moreover, in [1], there is an estimate

$$
\chi \geqslant 1+\frac{1}{\lambda_{\max }(L)-1},
$$

which coincides with the inequality $\chi \geqslant 1+\frac{1}{1-\lambda_{1}^{u p}}$ as above.

On a cyclical graph with $n$ vertices, Corollary 1 gives better estimate of the chromatic number than Hoffman's bound. For example, let's look at an odd cyclical graph, a ring with $(2 m+1)$ vertices, with $m \geqslant 2$. The largest Laplacian eigenvalue $\lambda_{\max }(L)=$ $1-\cos \frac{2 m \pi}{2 m+1}$, while $\mu_{1}^{u p}=1-\frac{2 m}{2 m+1}$. Then Hoffman's lower bound for chromatic number is $1-\frac{1}{\cos \frac{2 m \pi}{2 m+1}}$ which is smaller than our lower bound $\frac{2}{1-\mu_{1}^{u p}}=2+\frac{1}{m}$.

\subsection{Independence number and clique covering number for $d$-faces}

Similar to the results in [27], we give the counterpart of connections between the independence number and clique covering number of $K$ as well as the multiplicity of the eigenvalue 1 of $\Delta_{1}$. The definitions and notions are listed below.

- $\gamma$ : topological multiplicity of the maximal eigenvalue 1 of $\Delta_{1}$.

- $t$ : times of 1 appearing in the sequence of variational eigenvalues $\left(c_{k}\right)_{k=1}^{n}$.

- $\alpha$ : independence number of $S_{d}$, i.e., the cardinality of the largest subset of $d$-faces that does not adjacent to each other. It is defined by $\alpha=\max \{p$ : there exist $p$ faces in $S_{d}$ which are pairwise non-adjacent $\}$.

- $\kappa$ : the clique covering number (the smallest number of cliques of $S_{d}$ whose union covers $S_{d}$ ). Here a clique is a subset of $S_{d}$ such that any two $d$-faces in such clique are adjacent.

Theorem 13. The constants $\alpha, t, \gamma$ and $\kappa$ satisfy

$$
\alpha \leqslant t \leqslant \gamma \leqslant \kappa
$$


Proof. The inequality $t \leqslant \gamma$ is a basic result [5]. So it suffices to prove $\alpha \leqslant t$ and $\gamma \leqslant \kappa$.

$\alpha \leqslant t$ : We only need to show that $c_{k}=1$ whenever $k \geqslant n-\alpha+1$, where $n:=\# S_{d}$.

Since $n-k+1 \leqslant \alpha$, there exist $n-k+1$ non-adjacent $d$-faces in $S_{d}$, denoted by $i_{1}, \ldots, i_{n-k+1}$. Set $X_{n-k+1}=X \cap \operatorname{span}\left(e_{i_{1}}, \ldots, e_{i_{n-k+1}}\right)$, where $e_{1}=(1,0,0, \ldots, 0), e_{2}=$ $(0,1,0, \ldots, 0), \ldots, e_{n}=(0,0, \ldots, 0,1) \in \mathbb{R}^{n}$. For each $f$ in $X_{n-k+1}$, there exists some $\left(l_{1}, \ldots, l_{n-k+1}\right) \neq 0$ such that $f=l_{1} e_{i_{1}}+\cdots+l_{n-k+1} e_{i_{n-k+1}}$. It should be noted that $i \neq j$ if $f_{i} f_{j} \neq 0$. Thus, each nodal domain of $f$ must be a singleton set, which implies that $I(f)=1$. Therefore, it follows from $A \cap \operatorname{span}\left(e_{i_{1}}, \ldots, e_{i_{n-k+1}}\right) \subset X$ and the properties of genus that

$$
A \cap X_{n-k+1}=A \cap \operatorname{span}\left(e_{i_{1}}, \ldots, e_{i_{n-k+1}}\right) \cap X=A \cap \operatorname{span}\left(e_{i_{1}}, \ldots, e_{i_{n-k+1}}\right)
$$

is nonempty for any $A$ with $\gamma(A) \geqslant k$. Hence $\sup _{f \in A} I(f) \geqslant \inf _{f \in X_{n+1-k}} I(f)=1$ and then $c_{k}=1$.

$\gamma \leqslant \kappa$ : For a subset $W$ of $S_{d}$, let $I_{W}^{1}=\left\{f \in X: I(f)=1, f_{i}=0, \forall i \in S_{d} \backslash W\right\}$. Our purpose is to show $\gamma\left(I_{S_{d}}^{1}\right) \leqslant \kappa$. We first prove that for any disjoint subsets $W_{1}$ and $W_{2}$, $I_{W_{1} \cup W_{2}}^{1} \subset I_{W_{1}}^{1} * I_{W_{2}}^{1}$. Here $*$ is the topological join ${ }^{4}$. Given $a \in I_{W_{1} \cup W_{2}}^{1}$, let

$$
a_{i}^{1}=\left\{\begin{array}{ll}
a_{i}, & \text { if } i \in W_{1} \\
0, & \text { if } i \notin W_{1}
\end{array} \text { and } a_{i}^{2}=\left\{\begin{array}{ll}
a_{i}, & \text { if } i \in W_{2} \\
0, & \text { if } i \notin W_{2}
\end{array} \text { for } i \in V .\right.\right.
$$

Since $W_{1}$ and $W_{2}$ are disjoint, one can easily verify that $a=a^{1}+a^{2}$ and $1=I(a) \leqslant$ $I\left(a^{1}\right)+I\left(a^{2}\right) \leqslant\left\|a^{1}\right\|+\left\|a^{2}\right\|=\|a\|=1$. Hence, $I\left(a^{1}\right)=\left\|a^{1}\right\|$ and $I\left(a^{2}\right)=\left\|a^{2}\right\|$ hold. Taking $f=a^{1} /\left\|a^{1}\right\|$ and $g=a^{2} /\left\|a^{2}\right\|$, one has $I(f)=\|f\|=I(g)=\|g\|=1$, which implies that $f \in I_{W_{1}}^{1}$ and $g \in I_{W_{2}}^{1}$. Therefore, we have $a=\left\|a^{1}\right\| f+\left\|a^{2}\right\| g=t f+(1-t) g$, where $t=\left\|a^{1}\right\|$. Then we obtain

$$
I_{W_{1} \cup W_{2}}^{1} \subset\left\{t f+(1-t) g \mid f \in I_{W_{1}}^{1}, g \in I_{W_{2}}^{1}, t \in[0,1]\right\}=I_{W_{1}}^{1} * I_{W_{2}}^{1} .
$$

Combining the subadditivity of Krasnoselski genus with respect to topological join (see $[27])$, we have

$$
\gamma\left(I_{W_{1} \cup W_{2}}^{1}\right) \leqslant \gamma\left(I_{W_{1}}^{1} * I_{W_{2}}^{1}\right) \leqslant \gamma\left(I_{W_{1}}^{1}\right)+\gamma\left(I_{W_{2}}^{1}\right) .
$$

According to the mathematical induction, we can easily deduce that

$$
\gamma\left(I_{S_{d}}^{1}\right) \leqslant \min \left\{\sum_{i=1}^{l} \gamma\left(I_{W_{i}}^{1}\right): W_{1}, \ldots, W_{l} \text { form a partition of } S_{d}\right\},
$$

which provides a recurrence method to estimate a large complex by smaller one.

Note that for a clique $W$ of $S_{d}, I_{W}^{1}=\left\{f \in F: I(f)=1, f_{j} f_{l} \geqslant 0, \forall j, l \in W ; f_{i}=\right.$ $0, \forall i \notin W\}$. We can easily construct an odd continuous function $F: I_{W}^{1} \rightarrow \mathbb{S}^{0}=\{-1,1\}$ defined by

$$
F(f)= \begin{cases}1, & \text { if there exists } i \in W \text { such that } f_{i}>0 \\ -1, & \text { if there exists } i \in W \text { such that } f_{i}<0\end{cases}
$$

\footnotetext{
${ }^{4}$ The topological join of two sets $A$ and $B$ in a linear space is usually defined to be $A * B:=\{t a+(1-t) b$ : $\forall a \in A, b \in B, t \in[0,1]\}$.
} 
This means that $\gamma\left(I_{W}^{1}\right)=1$.

Then (15) implies that

$$
\begin{aligned}
\gamma\left(I_{S_{d}}^{1}\right) & \leqslant \min \left\{\sum_{i=1}^{l} \gamma\left(I_{W_{i}}^{1}\right) \text { : cliques } W_{1}, \ldots, W_{l} \text { form a partition of } S_{d}\right\} \\
& =\min \left\{l=\sum_{i=1}^{l} 1 \text { : cliques } W_{1}, \ldots, W_{l} \text { form a partition of } S_{d}\right\}=\kappa .
\end{aligned}
$$

Remark 14. Theorem 13 holds for both signless up 1-Laplacians and signless down 1Laplacians.

Remark 15. The inequality provided in Theorem 13 looks like the Lovász Sandwich theorem:

$$
\alpha \leqslant \Theta \leqslant \theta \leqslant \kappa
$$

where $\Theta$ is the Shannon capacity of a graph, and $\theta$ is the Lovász number (or Lovász theta function).

\section{Constructions and their effect on the spectrum}

Denote by $\operatorname{spec}(K)$ the set of all eigenvalues of $\Delta_{1}$. Considering the topological multiplicity, we use $\operatorname{Spec}(K)$ to denote the multiset of $\Delta_{1}$-eigenvalues. Furthermore, $A \cup B$ is the sum of two multisets $A$ and $B$. Our results are nonlinear analogs of the relevant theorems in [13], but most of the proofs are different.

\subsection{Wedges}

Definition 9. The combinatorial $k$-wedge sum $K_{1} \vee_{k} K_{2}$ of simplicial complexes $K_{1}$ and $K_{2}$, is defined as the quotient of their disjoint union by the identification $F_{1} \sim F_{2}$, that is

$$
K_{1} \vee_{k} K_{2}:=K_{1} \sqcup K_{2} /\left\{F_{1} \sim F_{2}\right\}
$$

where $F_{1}$ and $F_{2}$ are the $k$-dim simplicial faces in $K_{1}$ and $K_{2}$ respectively.

This definition could be generalized to the $k$-wedge sum of arbitrary many simplicial complexes. For example, the 1-wedge of some tetrahedrons is shown as below.

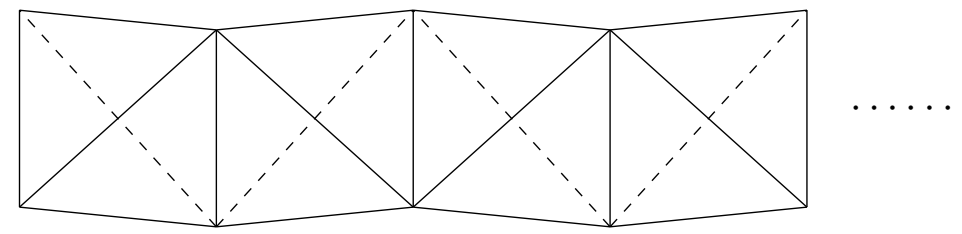

Theorem 16.

$$
\operatorname{Spec}\left(\Delta_{1, i}^{u p}\left(K_{1} \vee_{k} K_{2}\right)\right)=\operatorname{Spec}\left(\Delta_{1, i}^{u p}\left(K_{1}\right)\right) \stackrel{\circ}{\cup} \operatorname{Spec}\left(\Delta_{1, i}^{u p}\left(K_{2}\right)\right)
$$

for all $i, k$ with $0 \leqslant k<i$. 
Proof. Since $K_{1}$ and $K_{2}$ are identified by a $k$-face, then by noting that $k<i$, any $i$-face of $K_{1}$ is non-adjacent to $i$-faces of $K_{2}$ in $K_{1} \vee_{k} K_{2}$. Consequently, if $\left(\mu, f^{1}\right)$ is an eigenpair of $\Delta_{1, i}^{u p}\left(K_{1}\right)$, then letting

$$
f_{j}= \begin{cases}f_{j}^{1}, & j \in S_{i}\left(K_{1}\right), \\ 0, & j \in S_{i}\left(K_{2}\right),\end{cases}
$$

it is easy to check that $(\mu, f)$ is an eigenpair of $\Delta_{1, i}^{u p}\left(K_{1} \vee_{k} K_{2}\right)$. The same property holds for $\Delta_{1, i}^{u p}\left(K_{2}\right)$. Moreover, if $\left(\mu, f^{1}\right)$ and $\left(\mu, f^{2}\right)$ are eigenpairs of $\Delta_{1, i}^{u p}\left(K_{1}\right)$ and $\Delta_{1, i}^{u p}\left(K_{2}\right)$, respectively, then it can be easily verified that $(\mu, f)$ is an eigenpair of $\Delta_{1, i}^{u p}\left(K_{1} \vee_{k} K_{2}\right)$, where $f$ is defined by

$$
f_{j}= \begin{cases}f_{j}^{1}, & j \in S_{i}\left(K_{1}\right), \\ f_{j}^{2}, & j \in S_{i}\left(K_{2}\right) .\end{cases}
$$

So, we have proved that

$$
\operatorname{Spec}\left(\Delta_{1, i}^{u p}\left(K_{1} \vee_{k} K_{2}\right)\right) \supset \operatorname{Spec}\left(\Delta_{1, i}^{u p}\left(K_{1}\right)\right) \stackrel{\cup}{\cup} \operatorname{Spec}\left(\Delta_{1, i}^{u p}\left(K_{2}\right)\right)
$$

For the converse, let $(\mu, f)$ be an eigenpair of $\Delta_{1, i}^{u p}\left(K_{1} \vee_{k} K_{2}\right)$, and let $f^{1}$ (resp. $\left.f^{2}\right)$ be the restriction of $f$ on $S_{i}\left(K_{1}\right)$ (resp. $S_{i}\left(K_{2}\right)$ ). Since $f \neq 0$, at least one of $f^{1}$ and $f^{2}$ is not 0 . Suppose $f^{1} \neq 0$. Then, there exist $z_{j_{1} \cdots j_{i+1} j} \in \operatorname{Sgn}\left(f_{j_{1}}+\cdots+f_{j_{i+1}}+f_{j}\right)$ such that

$$
\sum_{j_{1}, \cdots, j_{i+1}} z_{j_{1} j_{2} \cdots j} \in \mu \operatorname{deg}_{j}^{u p} \operatorname{Sgn}\left(f_{j}\right)
$$

for any $j \in S_{i}\left(K_{1} \vee_{k} K_{2}\right)=S_{i}\left(K_{1}\right) \cup S_{i}\left(K_{2}\right)$. Therefore, for $j \in S_{i}\left(K_{1}\right)$, the above relation holds and thus $\left(\mu, f^{1}\right)$ is an eigenpair of $\Delta_{1, i}^{u p}\left(K_{1}\right)$. If $f^{2} \neq 0$, then the same process deduces that $\left(\mu, f^{2}\right)$ is an eigenpair of $\Delta_{1, i}^{u p}\left(K_{2}\right)$. Hence,

$$
\operatorname{Spec}\left(\Delta_{1, i}^{u p}\left(K_{1} \vee_{k} K_{2}\right)\right) \subset \operatorname{Spec}\left(\Delta_{1, i}^{u p}\left(K_{1}\right)\right) \stackrel{\cup}{\cup} \operatorname{Spec}\left(\Delta_{1, i}^{u p}\left(K_{2}\right)\right)
$$

The proof is completed.

Remark 17. This is a signless 1-Laplacian counterpart of Theorem 6.1 [13].

Similarly, we have the following

\section{Theorem 18.}

$$
\operatorname{Spec}\left(\Delta_{1, i}^{\text {down }}\left(K_{1} \vee_{k} K_{2}\right)\right)=\operatorname{Spec}\left(\Delta_{1, i}^{\text {down }}\left(K_{1}\right)\right) \stackrel{\circ}{\cup} \operatorname{Spec}\left(\Delta_{1, i}^{\text {down }}\left(K_{2}\right)\right)
$$

for all $i, k$ with $i>k+1$.

Theorem 19. Let $K_{1}$ and $K_{2}$ be simplicial complexes, for which the spectrum of $\Delta_{1, i}^{u p}\left(K_{1}\right)$ and $\Delta_{1, i}^{u p}\left(K_{2}\right)$ contain the eigenvalue $\mu$, and let $f^{1}, f^{2}$ be their corresponding eigenvectors. If an $i$-wedge $K=K_{1} \vee_{i} K_{2}$ is obtained by identifying $i$-faces $i_{1}$ and $i_{2}$, for which $f_{i_{1}}^{1}=f_{i_{2}}^{2}$, then the spectrum of $\Delta_{1, i}^{u p}(K)$ also contains the eigenvalue $\mu$. 
Proof. Note that we have identified $i_{1}$ with $i_{2}$ in $K$. So, we can assume $S_{i}\left(K_{1} \vee_{i} K_{2}\right)=$ $\left(S_{i}\left(K_{1}\right) \backslash\left\{i_{1}\right\}\right) \cup S_{i}\left(K_{2}\right)$. It is easy to see that

$$
\operatorname{deg}_{j}^{u p}(K)= \begin{cases}\operatorname{deg}_{j}^{u p}\left(K_{1}\right), & \text { if } j \in S_{i}\left(K_{1}\right) \backslash\left\{i_{1}\right\}, \\ \operatorname{deg}_{j}^{u p}\left(K_{2}\right), & \text { if } j \in S_{i}\left(K_{2}\right) \backslash\left\{i_{2}\right\}, \\ \operatorname{deg}_{i_{1}}^{u p}\left(K_{1}\right)+\operatorname{deg}_{i_{2}}^{u p}\left(K_{2}\right), & \text { if } j=i_{2} .\end{cases}
$$

Now we are going to prove that

$$
f_{j}= \begin{cases}f_{j}^{1}, & j \in S_{i}\left(K_{1}\right) \backslash\left\{i_{1}\right\} \\ f_{j}^{2}, & j \in S_{i}\left(K_{2}\right)\end{cases}
$$

is an eigenvector of $\Delta_{1, i}^{u p}(K)$ corresponding to the eigenvalue $\mu$. In fact, since $\left(\mu, f^{1}\right)$ is an eigenpair of $\Delta_{1, i}^{u p}\left(K_{1}\right)$, there exist $z_{j_{1} \cdots j_{i+1} j}^{1} \in \operatorname{Sgn}\left(f_{j_{1}}^{1}+\cdots+f_{j+1}^{1}+f_{j}^{1}\right)$ and $z_{j}^{1} \in \operatorname{Sgn}\left(f_{j}^{1}\right)$ such that

$$
\sum_{j_{1}, \ldots, j_{i+1}} z_{j_{1} j_{2} \cdots j_{i+1} j}^{1}=\mu \operatorname{deg}_{j}^{u p}\left(K_{1}\right) z_{j}^{1}
$$

for any $j \in S_{i}\left(K_{1}\right) \backslash\left\{i_{1}\right\}$, and

$$
\sum_{j_{1}, \ldots, j_{i+1}} z_{j_{1} j_{2} \cdots i_{1}}^{1}=\mu \operatorname{deg}_{i_{1}}^{u p}\left(K_{1}\right) z_{i_{1}}^{1} .
$$

Similarly, there exist $z_{j_{1} \cdots j_{i+1} j}^{2} \in \operatorname{Sgn}\left(f_{j_{1}}^{2}+\cdots+f_{j_{i+1}}^{2}+f_{j}^{2}\right)$ and $z_{j}^{2} \in \operatorname{Sgn}\left(f_{j}^{2}\right)$ such that

$$
\sum_{j_{1}, \ldots, j_{i+1}} z_{j_{1} j_{2} \cdots j}^{2}=\mu \operatorname{deg}_{j}^{u p}\left(K_{2}\right) z_{j}^{2}
$$

for any $j \in S_{i}\left(K_{2}\right)$. Now we take

$$
z_{j}= \begin{cases}z_{j}^{1}, & \text { if } j \in S_{i}\left(K_{1}\right) \backslash\left\{i_{1}\right\}, \\ z_{j}^{2}, & \text { if } j \in S_{i}\left(K_{2}\right) \backslash\left\{i_{2}\right\}, \\ \frac{\operatorname{deg}_{i_{1}}^{u p}\left(K_{1}\right)}{\operatorname{deg}_{i_{1}}^{u p}\left(K_{1}\right)+\operatorname{deg}_{i_{2}}^{u p}\left(K_{2}\right)} z_{i_{1}}^{1}+\frac{\operatorname{deg}_{i_{2}}^{u p}\left(K_{2}\right)}{\operatorname{deg}_{i_{1}}^{u p}\left(K_{1}\right)+\operatorname{deg}_{i_{2}}^{u p}\left(K_{2}\right)} z_{i_{2}}^{2}, & \text { if } j=i_{2},\end{cases}
$$

and

$$
z_{j_{1} j_{2} \cdots j_{i+1} j}= \begin{cases}z_{j_{1} j_{2} \cdots j_{i+1} j}^{1}, & \text { if } j \in S_{i}\left(K_{1}\right), \\ z_{j_{1} j_{2} \cdots j_{i+1} j}^{2}, & \text { if } j \in S_{i}\left(K_{2}\right) .\end{cases}
$$

Since $f_{i_{1}}^{1}=f_{i_{2}}^{2}$, then $\frac{\operatorname{deg}_{i_{1}}^{u p}\left(K_{1}\right)}{\operatorname{deg}_{i_{1}}^{u p}\left(K_{1}\right)+\operatorname{deg}_{i_{2}}^{u p}\left(K_{2}\right)} z_{i_{1}}^{1}+\frac{\operatorname{deg}_{i_{2}}^{u p}\left(K_{2}\right)}{\operatorname{deg}_{i_{1}}^{u p}\left(K_{1}\right)+\operatorname{deg}_{i_{2}}^{u p}\left(K_{2}\right)} z_{i_{2}}^{2} \in \operatorname{Sgn}\left(f_{i_{1}}^{1}\right)=\operatorname{Sgn}\left(f_{i_{2}}^{2}\right)=$ $\operatorname{Sgn}\left(f_{i_{2}}\right)$, which means that $z_{j}$ is well-defined. Finally, it can be easily verified that

$$
\sum_{j_{1}, \ldots, j_{i+1}} z_{j_{1} j_{2} \cdots j_{i+1} j}=\mu \operatorname{deg}_{j}^{u p}(K) z_{j}
$$

for any $j \in S_{i}(K)$, which completes the proof. 
Remark 20. This is a signless 1-Laplacian counterpart of Theorem 6.3 [13].

Theorem 21. Let $c_{1}, \ldots, c_{m}$ be the min-max eigenvalues of $\Delta_{1, i}^{u p}\left(K_{1} \cup K_{2}\right)$, and let $c_{1}^{\prime}$, $\ldots, c_{m-1}^{\prime}$ be the min-max eigenvalues of $\Delta_{1, i}^{u p}(K)$, where $K=K_{1} \vee_{i} K_{2}$, then

$$
c_{j} \leqslant c_{j}^{\prime}
$$

for every $1 \leqslant j \leqslant m-1$.

Proof. Let $i_{1}$ and $i_{2}$ be $i$-faces which are identified in an $i$-wedge sum $K$, which will be denoted by $i^{\prime}$ in $K$. Note that

$$
\begin{aligned}
I_{K_{1} \cup K_{2}}^{u p}(f)= & \sum_{j_{1}, \ldots, j_{i+2} \neq i_{1}, i_{2}}\left|f_{j_{1}}+\cdots+f_{j_{i+2}}\right|+\sum_{j_{2}, \cdots, j_{i+2}}\left|f_{i_{1}}+f_{j_{2}}+\cdots+f_{j_{i+2}}\right| \\
& +\sum_{j_{2}, \cdots, j_{i+2}}\left|f_{i_{2}}+f_{j_{2}}+\cdots+f_{j_{i+2}}\right|
\end{aligned}
$$

and

$$
I_{K}^{u p}(g)=\sum_{j_{1}, \ldots, j_{i+2} \neq i^{\prime}}\left|g_{j_{1}}+\cdots+g_{j_{i+2}}\right|+\sum_{j_{2}, \ldots, j_{i+2}}\left|g_{i^{\prime}}+g_{j_{2}}+\cdots+g_{j_{i+2}}\right|
$$

where all $j_{1}, \ldots, j_{i+2}$ under the sum notation are $i$-faces of a $(i+1)$-simplex. It is easy to see that

$$
\begin{aligned}
I_{K_{1} \cup K_{2}}^{u p}(f)-I_{K}^{u p}(g)= & \sum_{j_{2}, \ldots, j_{i+2}}\left|f_{i_{1}}+f_{j_{2}}+\cdots+f_{j_{i+2}}\right|+\sum_{j_{2}, \cdots, j_{i+2}}\left|f_{i_{2}}+f_{j_{2}}+\cdots+f_{j_{i+2}}\right| \\
& -\sum_{j_{2}, \cdots, j_{i+2}}\left|g_{i^{\prime}}+f_{j_{2}}+\cdots+f_{j_{i+2}}\right|
\end{aligned}
$$

whenever $g_{j}=f_{j}$ for $j \notin\left\{i^{\prime}, i_{1}, i_{2}\right\}$. And $I_{K_{1} \cup K_{2}}^{u p}(f)-I_{K}^{u p}(g)=0$ if we further assume that $g_{i^{\prime}}=f_{i_{1}}=f_{i_{2}}$.

Let

$$
X=\left\{f \in \mathbb{R}^{m}: \sum_{i=1}^{m} \operatorname{deg}_{i}^{u p}\left(K_{1} \cup K_{2}\right)\left|f_{i}\right|=1\right\}
$$

and

$$
Y=\left\{g \in \mathbb{R}^{m-1}: \sum_{i=1}^{m-1} \operatorname{deg}_{i}^{u p}(K)\left|g_{i}\right|=1\right\},
$$

where $m=\# S_{i}\left(K_{1}\right)+\# S_{i}\left(K_{2}\right)$. Note that

$$
\operatorname{deg}_{j}^{u p}(K)= \begin{cases}\operatorname{deg}_{i}^{u p}\left(K_{1} \cup K_{2}\right), & \text { if } j \in S_{i}(K) \backslash\left\{i^{\prime}\right\}, \\ \operatorname{deg}_{i_{1}}^{u p}\left(K_{1}\right)+\operatorname{deg}_{i_{2}}^{u p}\left(K_{2}\right), & \text { if } j=i^{\prime} .\end{cases}
$$

Let $\hat{X}=X \cap\left\{f \in \mathbb{R}^{m}: f_{i_{1}}=f_{i_{2}}\right\}$ and let $\psi: \hat{X} \rightarrow Y$ be defined by

$$
\psi(f)_{j}= \begin{cases}f_{j}, & \text { if } j \in S_{i}(K) \backslash\left\{i^{\prime}\right\} \\ f_{i_{2}}, & \text { if } j=i^{\prime}\end{cases}
$$


Then $\psi$ is an odd homeomorphism from $\hat{X}$ to $Y$. And $I_{K}^{u p}(\psi(f))=I_{K_{1} \cup K_{2}}^{u p}(f), \forall f \in \hat{X}$. Thus,

$$
\begin{aligned}
c_{j} & =\inf _{\gamma(A) \geqslant j, A \subset X} \sup _{f \in A} I_{K_{1} \cup K_{2}}^{u p}(f) \\
& \leqslant \inf _{\gamma(A) \geqslant j, A \subset \hat{X}} \sup _{f \in A} I_{K_{1} \cup K_{2}}^{u p}(f) \\
& =\inf _{\gamma(A) \geqslant j, A \subset \hat{X}} \sup _{f \in A} I_{K}^{u p}(\psi(f)) \\
& =\inf _{\gamma(B) \geqslant j, B \subset Y} \sup _{y \in B} I_{K}^{u p}(g)=c_{j}^{\prime} .
\end{aligned}
$$

The proof is completed.

\subsection{Duplication of motifs}

Given a simplicial complex $K=(V, S)$, let $M$ be a collection of faces. The closure $\mathrm{Cl} M$ of $M$ is the smallest subcomplex of $K$ that contains each simplex in $M$ and is obtained by repeatedly adding to $M$ each face of every simplex in $M$. The star St $M$ of $M$ is the set of all simplices in $K$ that have a face in $M$. The link lk $M$ of $M$ is $\mathrm{Cl}$ St $M \backslash \mathrm{St} \mathrm{Cl} M$.

Definition 10 (i-motif). A subcomplex $M$ of a simplicial complex $K$ is an $i$-motif if:

(1) $\left(\forall F_{1}, F_{2} \in M\right)$, if $F_{1}, F_{2} \subset F \in K$, then $F \in M$

(2) $\operatorname{dim} \operatorname{lk} M=i$.

From the definition of link, the vertices in motif $M$ are different from that in lk $M$. Let $l_{0}, \ldots, l_{m}$ be vertices of $1 \mathrm{k} M$ and $p_{0}, \ldots, p_{k}$ be the vertices of $M$. Duplication of the $i$-motif $M$ is defined as follows.

Definition 11 (duplication of the $i$-motif $M$ ). Let $M^{\prime}$ be a simplicial complex on the vertices $p_{0}^{\prime}, \ldots, p_{k}^{\prime}$ and let the map $h: p_{i}^{\prime} \rightarrow p_{i}$ be a simplicial isomorphism between $M^{\prime}$ and $M$. Let $K^{M}:=K \cup\left\{\left\{p_{i_{0}}^{\prime}, \ldots, p_{i_{k}}^{\prime}, l_{j_{1}}, \ldots, l_{j_{l}}\right\} \mid\left\{p_{i_{0}}, \ldots, p_{i_{k}}, l_{j_{1}}, \ldots, l_{j_{l}}\right\} \in K\right\}$. We call $K^{M}$ the duplication of $i$-motif of $M$.

Note that $K=(K-\mathrm{St} M) \vee_{i}(\mathrm{Cl}$ St $M)$. As a consequence of Theorem 16, we have the following

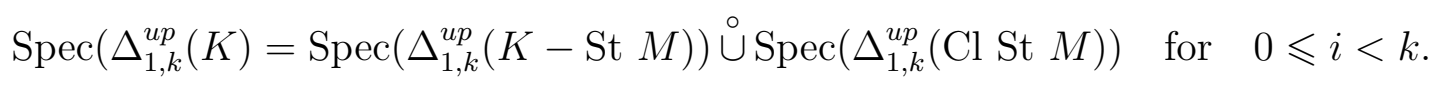

The following proposition can be proved by the similar methods in [13]. For completeness, we give the proof.

Proposition 4. For $i$-motif $M$, considering $\left.\Delta_{1, i}^{u p}(\mathrm{Cl} \mathrm{St} M)\right|_{\mathrm{St} M}$ which is the restriction of $\Delta_{1, i}^{u p}(\mathrm{Cl} \mathrm{St} M)$ on St $M$, if $(\mu, h)$ is the eigenpair of $\left.\Delta_{1, i}^{u p}(\mathrm{Cl} \mathrm{St} M)\right|_{\mathrm{St} M}$, then $(\mu, \varrho)$ is an eigenpair of $\Delta_{1, i}^{u p}\left(K^{M}\right)$, where

$$
\varrho(F)= \begin{cases}h(F), & \text { if } F \in \text { St } M \\ -h(F), & \text { if } F \in \text { St } M^{\prime} \\ 0, & \text { otherwise }\end{cases}
$$


Proof. According to the definition of St $M$, for any $F \in$ St $M$, if $F \subset \bar{F}$, then $\bar{F} \in$ St $M$, which implies that $\Delta_{1, i}^{u p}(\mathrm{Cl}$ St $M)$ and $\Delta_{1, i}^{u p}\left(K^{M}\right)$ coincide on St $M$ and for any $i$-face $F$ in St $M$, there is $\operatorname{deg}_{F}^{u p}(\mathrm{Cl}$ St $M)=\operatorname{deg}_{F}^{u p}\left(K^{M}\right)$. Let

$$
\varrho(F)= \begin{cases}h(F), & \text { if } F \in \text { St } M \\ -h(F), & \text { if } F \in \text { St } M^{\prime} \\ 0, & \text { otherwise }\end{cases}
$$

Then,

$$
\Delta_{1, i}^{u p}\left(K^{M}\right) \varrho(F)= \begin{cases}\left.\Delta_{1, i}^{u p}(\mathrm{Cl} \mathrm{St} M)\right|_{\text {St } M} h(F) \in \mu \operatorname{deg}_{F}^{u p} \operatorname{Sgn}(\varrho(F)), & \text { for } \forall F \in \text { St } M ; \\ \left.\Delta_{1, i}^{u p}(\operatorname{Cl~St~} M)\right|_{\text {St } M}(-h(F)) \in \mu \operatorname{deg}_{F}^{u p} \operatorname{Sgn}(\varrho(F)), & \text { for } \forall F \in \text { St } M^{\prime} ; \\ 0 \in \mu \operatorname{deg}_{F}^{u p} \operatorname{Sgn}(\varrho(F)), & \text { otherwise, }\end{cases}
$$

which confirms the claim.

Similar to [13], we have the following corollary.

Corollary 2. If the spectrum of the simplicial complex $\mathrm{Cl}$ St $M$ contains the eigenvalue $\mu$, with an eigenvector $h$ that is zero on lk $M$, then $\mu$ is also the eigenvalue of $K^{M}$.

Using the same methods in the proof of Theorem 21, we have the following

Theorem 22. Let $c_{j}$ be the min-max eigenvalue of $\left.\Delta_{1, i}^{u p}(C l S t M)\right|_{S t M}$ and $c_{j}^{\prime}$ be the minmax eigenvalue of $\Delta_{1, i}^{u p}(C l$ St $M)$. Then $c_{j}^{\prime} \leqslant c_{j}$.

Proof. Let $X=\left\{f \in\right.$ St $\left.M: \sum_{f_{i} \in \mathrm{St} M} \operatorname{deg}_{i}^{u p}\left|f_{i}\right|=1\right\}$ and $Y=\{g \in$ Cl St $M$ : $\left.\sum_{g_{j} \in \mathrm{Cl} \mathrm{St} M} \operatorname{deg}_{j}^{u p}\left|g_{j}\right|=1\right\}$ and $\widehat{Y}=Y \cap\left\{f: f_{m}=0, \forall f_{m} \in \mathrm{Cl}\right.$ St $M \backslash$ St $\left.M\right\}$. It is obvious that $F: \widehat{Y} \rightarrow X$ defined by

$$
F(f)_{j}= \begin{cases}f_{j}, & \text { if } f_{j} \in \mathrm{St} M \\ 0, & \text { if } f_{j} \in \mathrm{Cl} \mathrm{St} M \backslash \text { St } M,\end{cases}
$$

is an odd homemorphism. Then,

$$
\begin{aligned}
c_{j}^{\prime} & =\inf _{\gamma(A) \geqslant j, A \subset Y} \sup _{f \in A} I_{\mathrm{Cl} \mathrm{St} M}^{u p}(f) \\
& \leqslant \inf _{\gamma(A) \geqslant j, A \subset \widehat{Y}} \sup _{f \in A} I_{\mathrm{Cl} \text { St } M}^{u p}(f) \\
& =\inf _{\gamma(A) \geqslant j, A \subset \widehat{Y}} \sup _{f \in A} I_{\mathrm{St} M}^{u p}(F(f)) \\
& =\inf _{\gamma(B) \geqslant j, B \subset X} \sup _{g \in B} I_{\mathrm{St} M}^{u p}(g) \\
& =c_{j} .
\end{aligned}
$$

Remark 23. Theorem 21 and Theorem 22 are counterparts of Theorem 6.4 and Theorem 6.12 in [13] respectively. 


\section{Acknowledgements}

The authors are supported by grant from the Project funded by China Postdoctoral Science Foundations (No. BX201700009 and No. 2019M660829). We thank the referee for detailed suggestions on the former version of the paper, which improve the presentation of the paper.

\section{References}

[1] Naneh Apkarian, Spectral Bounds on the Chromatic Number, 2013

[2] Christine Bachoc, Anna Gundert and Alberto Passuello, The theta number of simplicial complexes, Israel Journal of Mathematics, 232 (2019), 443-481.

[3] Frank H Clarke, Optimization and Nonsmooth Analysis, Wiley New York, 1983.

[4] Kung-Ching Chang, Sihong Shao and Dong Zhang, Spectrum of the signless 1Laplacian and the dual Cheeger constant on graphs, arXiv:1607.00489.

[5] Kung-Ching Chang, Spectrum of the 1-laplacian and Cheeger's constant on graphs, Journal of Graph Theory, 81 (2016), 167-207.

[6] Kung-Ching Chang, Sihong Shao and Dong Zhang, The 1-Laplacian Cheeger cut: theory and algorithms, Journal of Computational Mathematics, 33 (2015), 443-467.

[7] Kung-Ching Chang, Sihong Shao and Dong Zhang, Nodal domains of eigenvectors for 1-Laplacian on graphs, Advances in Mathematics, 308 (2017), 529-574.

[8] Kung-Ching Chang, Critical Point Theory and Its Applications (in Chinese), Shanghai Science and Technology Press, 1985.

[9] Shai Evra, Konstantin Golubev and Alexander Lubotzky, Mixing properties and the chromatic number of ramanujan complexes. International Mathematics Research Notices, (2015) 11520-11548.

[10] Beno Eckmann, Harmonische funktionen und randwertaufgaben in einem komplex, Commentarii Mathematici Helvetici, 17 (1944), 240-255.

[11] Konstantin Golubev, On the chromatic number of a simplicial complex. Combinatorica, 37 (2017), 953-964.

[12] Danijela Horak and Jürgen Jost, Interlacing inequalities for eigenvalues of discrete Laplace operators, Annals of Global Analysis and Geometry, 43 (2013), 177-207.

[13] Danijela Horak and Jürgen Jost, Spectra of combinatorial Laplace operators on simplicial conplexes, Advances in Mathematics, 244 (2013), 303-336.

[14] Alan J Hoffman, On eigenvalues and colorings of graphs, b. harris ed., graph theory and its applications, 1970.

[15] Alan J Hoffman, On eigenvalues and colorings of graphs. In Selected Papers Of Alan J Hoffman: With Commentary, pages 407-419. World Scientific, 2003. 
[16] Matthias Hein and Thomas Bühler, An inverse power method for nonlinear eigenproblems with applications in 1-spectral clustering and sparse PCA. In Advances in Neural Information Processing Systems 23, pages 847-855, 2010.

[17] Tali Kaufman and Alexander Lubotzky, High dimensional expanders and property testing. In Proceedings of the 5th conference on Innovations in theoretical computer science, pages 501-506, 2014.

[18] Tali Kaufman and Izhar Oppenheim, High order random walks: Beyond spectral gap. arXiv: 1707.02799, 2017.

[19] Michael Krivelevich and Benny Sudakov, The chromatic numbers of random hypergraphs, Random Structures \& Algorithms, 12 (1998), 381-403.

[20] Alexander Lubotzky, Ramanujan complexes and high dimensional expanders, Japanese Journal of Mathematics, 9 (2014), 137-169.

[21] Alexander Lubotzky, High dimensional expanders. arXiv:1712.02526.

[22] Alexander Lubotzky and Ori Parzanchevski, From ramanujan graphs to ramanujan complexes. Philosophical Transactions of the Royal Society A, 378(2163):20180445, 2020.

[23] Vladimir Nikiforov, Hoffman's bound for hypergraphs. arXiv:1908.01433, 2019.

[24] Ori Parzanchevski and Ron Rosenthal, Simplicial complexes: spectrum, homology and random walks. Random Structures \& Algorithms, 50 (2017), 225-261.

[25] Paul Henry Rabinowitz, Minimax Methods in Critical Point Theory with Applications in Differential Equations, American Mathematical Society, 1986.

[26] Pawel Wocjan and Clive Elphick, New Spectral Bounds on the Chromatic Number Encompassing all Eigenvalues of the Adjacency Matrix, The Electronic Journal of Combinatorics, 20(3) \#P39 (2013).

[27] Dong Zhang, Topological multiplicity of the maximum eigenvalue of graph 1Laplacian, Discrete Mathematics, 341 (2018), 25-32. 